\title{
Isotopic abundance of nitrogen and carbon in distant comets ${ }^{\star}$
}

\author{
J. Manfroid ${ }^{1, \star \star}$, E. Jehin ${ }^{2}$, D. Hutsemékers ${ }^{1, \star \star \star}$, A. Cochran ${ }^{3}$, J.-M. Zucconi ${ }^{4}$, C. Arpigny ${ }^{1}$, \\ R. Schulz ${ }^{5}$, and J. A. Stüwe ${ }^{6}$
}

${ }^{1}$ Institut d'Astrophysique et de Géophysique, Université de Liège, Allée du 6 Août 17, 4000 Liège, Belgium e-mail: manfroid@astro.ulg.ac.be

2 European Southern Observatory, Casilla 19001, Santiago, Chile

3 Department of Astronomy and McDonald Observatory, University of Texas at Austin, C-1400 Austin, USA

4 Observatoire de Besançon, 25010 Besançon Cedex, France

5 ESA/RSSD, ESTEC, PO Box 299, 2200 AG Noordwijk, The Netherlands

${ }^{6}$ Leiden Observatory, 2300 RA Leiden, The Netherlands

Received 13 December 2004 / Accepted 20 January 2005

\begin{abstract}
The ${ }^{12} \mathrm{C}^{14} \mathrm{~N} /{ }^{12} \mathrm{C}^{15} \mathrm{~N}$ and ${ }^{12} \mathrm{C}^{14} \mathrm{~N} /{ }^{13} \mathrm{C}^{14} \mathrm{~N}$ isotopic ratios have been determined in comets $\mathrm{C} / 1995$ O1 (Hale-Bopp), C/2001 Q4 (NEAT) and C/2003 K4 (LINEAR) at heliocentric distances of, respectively, 2.7, 3.7 and 2.6 AU. These ratios have also been measured at $r \sim 1$ AU. No significant differences were found between all determinations, nor with the value obtained for other comets. If confirmed, the discrepancy between the nitrogen isotopic ratios from optical and millimeter measurements on $\mathrm{CN}$ and $\mathrm{HCN}$ would rule out $\mathrm{HCN}$ as a major parent of the cometary $\mathrm{CN}$ radicals.
\end{abstract}

Key words. comets: abundances - comets: individual: C/1995 O1 (Hale-Bopp), C/2001 Q4 (NEAT), C/2003 K4 (LINEAR)

\section{Introduction}

Determination of the abundance ratios of the stable isotopes of the light elements in different objects of the solar system provides important clues in the study of its origin and early history. Comets carry most valuable information regarding the material in the primitive solar nebula.

We identified for the first time a number of emission features belonging to the ${ }^{12} \mathrm{C}^{15} \mathrm{~N} \mathrm{~B}-\mathrm{X}(0,0)$ band in spectra obtained with the SOFIN spectrograph at the Nordic Optical Telescope (Canary Islands). This allowed us to make the first optical measurement of the nitrogen isotopic ratio ${ }^{14} \mathrm{~N} /{ }^{15} \mathrm{~N}$ in a comet (Arpigny et al. 2003). This ratio was found to be lower by a factor of about two than the terrestrial value (272) and, less than half those obtained in Hale-Bopp from millimiter measurements of $\mathrm{HCN}$, a possible main parent of $\mathrm{CN}\left({ }^{14} \mathrm{~N} /{ }^{15} \mathrm{~N}=323 \pm 46\right.$ at $r=1.2$ AU: Jewitt et al. 1997, $330 \pm 98, r=0.92$ AU: Ziurys et al. 1999). Spectra of fainter comets obtained with the ESO VLT and the $2.7 \mathrm{~m} \mathrm{McDonald}$ telescopes gave similar results, viz $140 \pm 25$ and $170 \pm 50$ for comets $122 \mathrm{P} / \mathrm{de}$ Vico $(r=0.66 \mathrm{AU})$ and 153P/IkeyaZhang ( $r=0.92 \mathrm{AU}$ ) respectively (Jehin et al. 2004). On the other hand the optical determinations of the ${ }^{12} \mathrm{C} /{ }^{13} \mathrm{C}$ ratio

* Based on observations collected at the European Southern Observatory, Paranal, Chile (ESO Programmes 270.C-5043, 073.C-0525 and 274.C-5015).

$\star \star$ Research Director FNRS.

$\star \star \star$ Research Associate FNRS. consistently yield values around 90, only slightly lower than the HCN millimiter measurements (respectively $111 \pm 12$ and $109 \pm 22$ from the above-cited authors), and in agreement with the solar value (89).

The differing results for nitrogen in $\mathrm{CN}$ and $\mathrm{HCN}$ would indicate that cometary $\mathrm{CN}$ radicals are produced from at least one other source with a much lower $\mathrm{N}$ isotopic ratio.

A monitoring of Hale-Bopp showed that somewhere beyond about $3 \mathrm{AU}$, the production rates of $\mathrm{HCN}$ and $\mathrm{CN}$ agree with one another within certain limits (Rauer et al. 2003). Hence, sufficiently far away from the Sun, HCN could be the only source of $\mathrm{CN}$ such that ${ }^{14} \mathrm{~N} /{ }^{15} \mathrm{~N}$ from $\mathrm{CN}$ should match the $\mathrm{HCN}$ value. In order to investigate this possibility, we measured the $\mathrm{N}$ isotopic ratio in three comets at such heliocentric distances. By comparison, our previously published data concern comets much closer to the Sun (0.6-1.3 AU).

\section{Observations and data analysis}

Comets C/1995 O1 (Hale-Bopp), C/2001 Q4 (NEAT) and C/2003 K4 (LINEAR) have been observed at heliocentric distances of, respectively, 2.7, 3.7 and 2.6 AU, and all three were also observed close to perihelion. Various telescopes were used in Chile, the USA, and the Canary Islands. Spectra of the $\mathrm{CN}$ violet band were obtained at resolving power of 60000 or higher (see Table 1).

The techniques used to reduce the data and measure the isotopic ratios are described in Arpigny et al. (2003) and 
Table 1. Observational data. $r$ is the heliocentric distance. $m_{\mathrm{r}}$ is the heliocentric visual magnitude. $2 \mathrm{dc}$ is the McDonald 2D-coude spectrograph, SOFIN and UVES are the high-resolution spectrographs at, respectively, the NOT and ESO VLT telescopes. $n$ is the number of spectra obtained, for a total exposure time of Exp. Extent is the size of the zone explored at the distance of the comet. $R$ is the instrumental resolving power. Fwhm is the measured width of the $\mathrm{CN}$ lines.

\begin{tabular}{|c|c|c|c|c|c|c|c|c|c|c|c|}
\hline & Date & Comet & $\begin{array}{c}r \\
(\mathrm{AU}) \\
\end{array}$ & $\begin{array}{c}\dot{r} \\
(\mathrm{~km} / \mathrm{s}) \\
\end{array}$ & $m_{\mathrm{r}}$ & Instr. & $n$ & $\begin{array}{l}\text { Exp. } \\
\text { (ks) }\end{array}$ & $\begin{array}{c}\text { Extent } \\
(\mathrm{km})\end{array}$ & $R$ & $\begin{array}{c}\text { Fwhm } \\
(\AA)\end{array}$ \\
\hline 1 & Oct96 & \multirow{3}{*}{ C/1995 O1 (Hale-Bopp) } & 2.73 & -20.7 & 3.0 & $2 \mathrm{dc}$ & 17 & 30.6 & 10000 & 60000 & 0.079 \\
\hline 2 & Apr97 & & 0.92 & 3.0 & -1.3 & $2 \mathrm{dc}$ & 3 & 2.8 & 2000 & 190000 & 0.040 \\
\hline 3 & Apr97 & & 0.93 & $4.0: 7.5$ & -1.3 & SOFIN & 23 & 32.5 & 20000 & 150000 & 0.053:0.066 \\
\hline 4 & Sep03 & \multirow{2}{*}{ C/2001 Q4 (NEAT) } & $3.73: 3.66$ & -18.8 & 9.5 & UVES & 4 & 23.4 & 20000 & 80000 & 0.0505 \\
\hline 5 & May04 & & 0.98 & $-5.4:-4.8$ & 5.6 & UVES & 11 & 20.7 & 50000 & 80000 & 0.052 \\
\hline 6 & May04 & \multirow{2}{*}{ C/2003 K4 (LINEAR) } & 2.61 & -20.3 & 9.2 & UVES & 1 & 4.4 & 7000 & 70000 & 0.056 \\
\hline 7 & Nov04 & & 1.20 & 14.7 & 6.3 & UVES & 2 & 3.0 & 5000 & 80000 & 0.050 \\
\hline
\end{tabular}

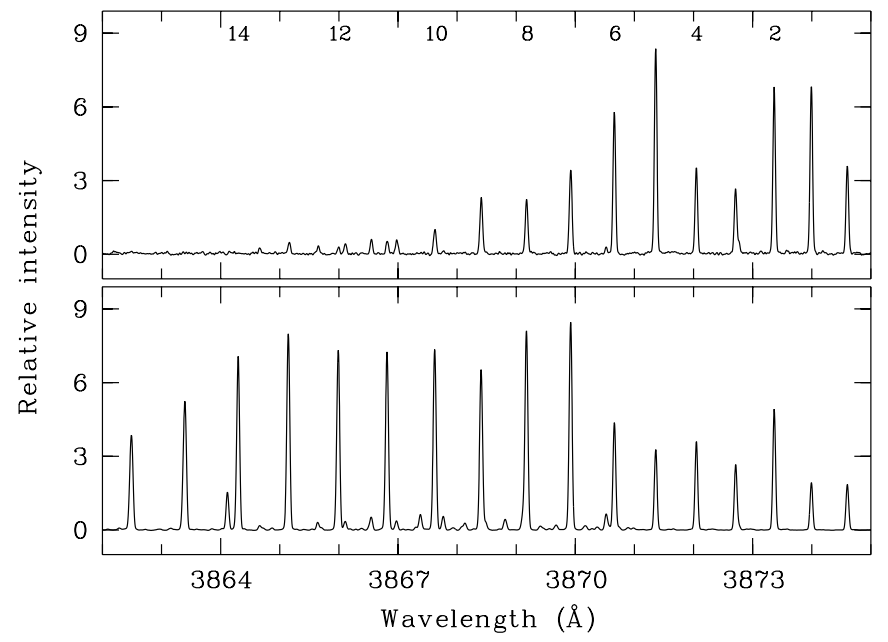

Fig. 1. Spectra of comet C/2001 Q4 at 3.7 AU (top) and 0.98 AU showing part of the $\mathrm{R}$ branch of the $\mathrm{CN} \mathrm{B}-\mathrm{X}(0,0)$ band. The corresponding quantum numbers are indicated at the top. The major differences are related to the heliocentric distance and show up in the excitation of lines of higher quantum number when closer to the Sun. The intensity scale is relative only.

Jehin et al. (2004). The ${ }^{12} \mathrm{C} /{ }^{13} \mathrm{C}$ and ${ }^{14} \mathrm{~N} /{ }^{15} \mathrm{~N}$ ratios are obtained simultaneously by fitting the spectra with theoretical models. An empirical correction was adopted to take account of additional effects not covered by the pure fluorescence model (collisional and possibly other unidentified effects).

Indeed, the comparison of theoretical and observational ${ }^{12} \mathrm{C}^{14} \mathrm{~N}$ data for several comets shows that a 3-parameter function, with the quantum number, $N$, as the independant variable, always brings a satisfactory agreement. The intensity $f_{N}$ of line $N$ is corrected in the following way:

$$
F_{N}=f_{N} \mathrm{e}^{c N} /\left(\mathrm{e}^{(N-a) / b}+1\right)
$$

with $a, b, c$ being estimated through an iterative process, for each individual spectrum. The same correction is applied to ${ }^{13} \mathrm{C}^{14} \mathrm{~N}$ and ${ }^{12} \mathrm{C}^{15} \mathrm{~N}$ under the assumption that these spectra are similarly affected. These corrections typically stay within $15 \%$. In cases of severe collision effects, in the central region of the
Table 2. The carbon and nitrogen isotopic ratios, and the corresponding estimated errors in parentheses.

\begin{tabular}{|c|c|c|c|}
\hline \multicolumn{2}{|r|}{ Comet } & \multirow{2}{*}{$\frac{{ }^{12} \mathrm{C} /{ }^{13} \mathrm{C}}{80(20)}$} & \multirow{2}{*}{$\frac{{ }^{14} \mathrm{~N}^{15} \mathrm{~N}}{140(30)}$} \\
\hline 1 & \multirow{3}{*}{ C/1995 O1 (Hale-Bopp) } & & \\
\hline 2 & & $90(30)$ & $160(40)$ \\
\hline 3 & & $95(40)$ & $140(45)$ \\
\hline 4 & \multirow{2}{*}{ C/2001 Q4 (NEAT) } & $70(30)$ & $130(40)$ \\
\hline 5 & & $90(15)$ & $135(20)$ \\
\hline 6 & \multirow{2}{*}{ C/2003 K4 (LINEAR) } & $85(20)$ & $150(35)$ \\
\hline 7 & & $90(15)$ & $135(20)$ \\
\hline
\end{tabular}

coma of $\mathrm{C} / 2001 \mathrm{Q} 4$, they can reach up to $40 \%$. One spectrum taken on May 6, 2004, right on the nucleus shows corrections of from $-65 \%$ at $R(0)$ up to $+65 \%$ around $R(15)$. The rms deviation of the fit between the observations and the corrected model is generally between 5 and $15 \%$, i.e., 3 to 4 times smaller than for the uncorrected model.

Only the R lines of the B - X $(0,0)$ band (i.e., shortward of $3875 \AA$ ) are used since the $\mathrm{P}$ lines of the three isotopes are strongly blended. The spectra obtained at large heliocentric distance have relatively low $\mathrm{S} / \mathrm{N}$ ratios. This makes the use of a global fitting technique mandatory. As seen in Fig. 1 the bulk of the $\mathrm{CN}$ emission is concentrated in fewer lines, namely those of low quantum number, when farther from the Sun. This increases the overall $\mathrm{S} / \mathrm{N}$ ratio for the isotopic lines since the noise is dominated by the background and the CCD readout. Simultaneously, the low-excitation spectra are less contaminated by the $\mathrm{P}$ lines of the $(1,1)$ band of the dominant species.

The estimated isotopic ratios are presented in Table 2 and plotted versus the heliocentric distance in Fig. 2. The values based on the Hale-Bopp SOFIN observations are slightly different from (and supersede) those given by Arpigny et al. (2003). This is mainly due to the use of better molecular parameters in the fluorescence model and of the ad-hoc empirical 


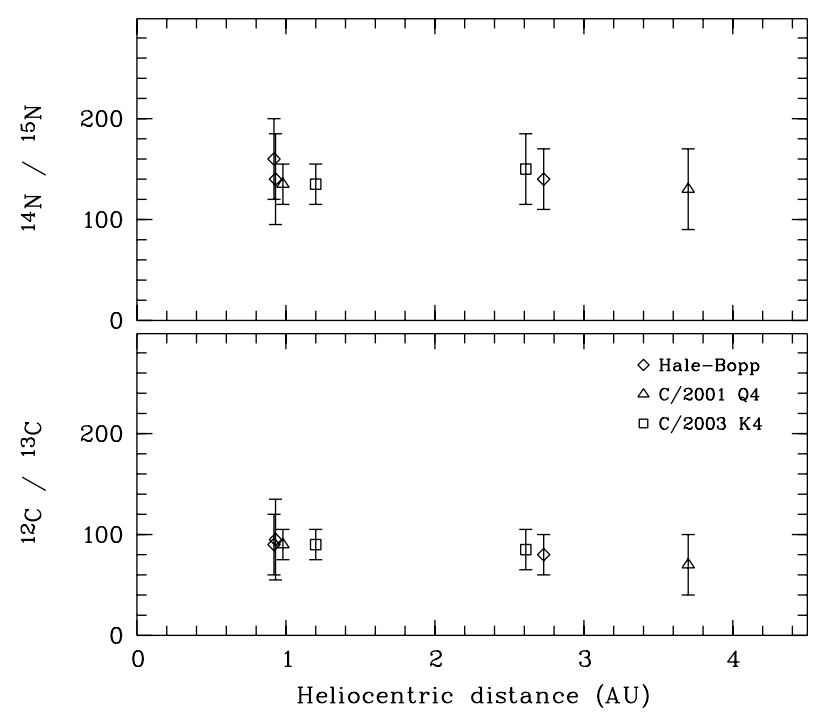

Fig. 2. Nitrogen and carbon isotopic ratios at various heliocentric distances.

correction. The Hale-Bopp SOFIN values agree with the ratios from McDonald spectra obtained quasi simultaneously.

\section{Discussion}

Our measurements show that changes in the ${ }^{14} \mathrm{~N} /{ }^{15} \mathrm{~N}$ and ${ }^{12} \mathrm{C} /{ }^{13} \mathrm{C}$ ratios with heliocentric distance are small as determined from the $\mathrm{CN}$ radical. This means that the $\mathrm{CN}$ isotopic mixture remains constant within the explored range of heliocentric distances, and is the same for every comet, with average values of, respectively, 86 and 141 for ${ }^{12} \mathrm{C} /{ }^{13} \mathrm{C}$ and ${ }^{14} \mathrm{~N} /{ }^{15} \mathrm{~N}$. The carbon ratio is compatible with previous measurements, including millimiter measurements in $\mathrm{HCN}$. On the other hand the nitrogen ratio is compatible with optical measurements of $\mathrm{CN}$ in other comets, but not with the $\mathrm{HCN}$ observations of Hale-Bopp.

In order to get some idea of the constraints on the actual isotopic variations, we assume two sources of $\mathrm{CN}$, namely $\mathrm{HCN}$ and $X$. Let us call $\zeta=\left[{ }^{12} \mathrm{C}^{14} \mathrm{~N} /{ }^{12} \mathrm{C}^{15} \mathrm{~N}\right]_{\text {total }}$ the observed isotopic ratio of $\mathrm{CN}$, and $\zeta_{\mathrm{H}}=\left[{ }^{12} \mathrm{C}^{14} \mathrm{~N} /{ }^{12} \mathrm{C}^{15} \mathrm{~N}\right]_{\mathrm{HCN}}$ the isotopic ratio of the $\mathrm{CN}$ radicals coming from $\mathrm{HCN}$. Similarly, we define $\zeta_{\mathrm{X}}=\left[{ }^{12} \mathrm{C}^{14} \mathrm{~N} /{ }^{12} \mathrm{C}^{15} \mathrm{~N}\right]_{\mathrm{X}}$.

We call $\epsilon=\mathrm{CN}_{\mathrm{HCN}} / \mathrm{CN}_{\text {total }} \approx{ }^{12} \mathrm{C}^{14} \mathrm{~N}_{\mathrm{HCN}} /{ }^{12} \mathrm{C}^{14} \mathrm{~N}_{\text {total }}$ the fraction of $\mathrm{CN}$ coming from $\mathrm{HCN}$.

One has

$1 / \zeta \approx \epsilon\left(1 / \zeta_{\mathrm{H}}-1 / \zeta_{\mathrm{X}}\right)+1 / \zeta_{\mathrm{X}}$

so that

$\left(1 / \zeta_{1}-1 / \zeta_{2}\right) \approx\left(\epsilon_{1}-\epsilon_{2}\right)\left(1 / \zeta_{\mathrm{H}}-1 / \zeta_{\mathrm{X}}\right)$

where subscripts 1 and 2 refer to different observations - e.g., the same comet at different heliocentric or nucleocentric distances, or different comets. Hence, assuming that $\zeta_{\mathrm{X}}$ and $\zeta_{\mathrm{H}}$ do not vary, changes in the observed ratio $1 / \zeta={ }^{12} \mathrm{C}^{15} \mathrm{~N} /{ }^{12} \mathrm{C}^{14} \mathrm{~N}$ are directly proportional to $\epsilon_{1}-\epsilon_{2}$, i.e., to changes in the relative importance of the column density of $\mathrm{CN}_{\mathrm{HCN}}$ or, with the opposite sign, of $\mathrm{CN}_{\mathrm{X}}$.

Let us consider an isotopic mixture with the observed ratio $\zeta_{1}=140$ and an isotopic ratio $\zeta_{\mathrm{H}}=325$ corresponding to the millimiter observations of HCN. Equation (2) links $\zeta_{\mathrm{X}}$ and $\epsilon_{1}$. Equation (3) can be used to calculate the proportion $\epsilon_{2}$ needed to produce a significant change in the observed ratio, say $\zeta_{2}=200$, which would be easily detected.

First, we assume that $\zeta_{1}=140$ characterizes a mixture of $\mathrm{CN}_{\mathrm{HCN}}$ and $\mathrm{CN}_{\mathrm{X}}$ in equal proportion $\left(\epsilon_{1}=0.5\right)$. The isotopic ratio of $\mathrm{X}$ is then $\zeta_{\mathrm{X}}=89$. The proportion of $\mathrm{CN}_{\mathrm{HCN}}$ must increase to $\epsilon_{2}=0.76$ in order to yield an overall $\zeta_{2}=200$.

As another (extreme) example, let us assume that the observed overall $\zeta_{1}=140$ characterizes $\mathrm{CN}$ coming exclusively from $X\left(\epsilon_{1}=0\right)$. The ${ }^{14} \mathrm{~N} /{ }^{15} \mathrm{~N}$ ratio $\zeta_{2}=200$ is reached when $53 \%$ of $\mathrm{CN}$ comes from $\mathrm{HCN}\left(\epsilon_{2}=0.53\right)$.

Our observations show that there is no such change in composition up to $3.7 \mathrm{AU}$.

Then, the hypothetical transition zone between a pure $\mathrm{HCN}$ source and a multiple source of $\mathrm{CN}$ must be farther out than 3.7 AU. This does not fit well with Rauer et al.'s (2003) results.

From Eq. (3), imposing the constancy of the isotopic ratios measured in several comets at various distances $\left(\zeta_{1}=\zeta_{2}\right)$, we find:

(i) $\zeta_{\mathrm{X}}=\zeta_{\mathrm{H}}=\zeta(=140)$, isotopically homogeneous sources, no constraints on the mixture proportions; or

(ii) $\epsilon_{1}=\epsilon_{2}$, the mixtures have identical proportions.

We shall not discuss further hypothesis (ii) because a highly improbable conjunction of coincidences would be needed to achieve the constancy of the fractions $\epsilon$ in every comet, under any conditions.

The first hypothesis (i) implies that either the observed $\mathrm{HCN}$ or $\mathrm{CN}$ nitrogen isotopic ratio is faulty, or that the observed $\mathrm{CN}$ does not come from $\mathrm{HCN}$.

In the optical domain, the measurement of the $\mathrm{CN}$ isotopic ratios is straightforward as soon as the $\mathrm{S} / \mathrm{N}$ ratio is high enough. The number of lines involved (a minimum of 7 in the present analysis) makes the possible influence of underlying unidentified features rather improbable. The fluorescence models appear to be reliable, so errors by a factor 2 seem to be excluded.

The HCN isotopic ratio is difficult to measure, and so far has been obtained in only one comet, the exceptionally bright Hale-Bopp, but the two independent measurements agree with one another and there is no reason to discard them. If there was a problem, the carbon ratio should probably show it in similar proportions. Nevertheless, it would be important to see whether the HCN results can be confirmed on a broad sample of comets in order to pin-point the nature of the problem of the isotopic ratio of $\mathrm{N}$. Are there actually large $\mathrm{N}$ isotopic differences between the various constituents of a comet? Or does the cometary material have an overall excess of ${ }^{15} \mathrm{~N}$ as compared to our current estimates of the solar nebula? Did the problem arise in this primitive nebula or is it linked to the formation and history of the cometary bodies? 
The discrepancy between the observed $\mathrm{CN}$ and $\mathrm{HCN}$ isotopic ratios could be explained if most of the $\mathrm{CN}$ released by $\mathrm{HCN}$ is produced outside of the zones explored by the observations, i.e., farther than about $5 \times 10^{4} \mathrm{~km}$ from the nucleus. $\mathrm{HCN}$ would then photodissociate slowly, much farther than expected from the nucleus. Its contribution to $\mathrm{CN}$ along the line of sight toward more central regions of the coma would be negligible. A sufficiently low dissociation rate would make the corresponding $\mathrm{CN}$ invisible even at large heliocentric distances. However, the spatial distribution of $\mathrm{HCN}$ is rather steep and excludes such large scale lengths (see, e.g. Irvine et al. 1998). The major sources of $\mathrm{CN}-$ at small and large heliocentric distances must then be some other molecule(s) with a dissociation scale length rather comparable to that of $\mathrm{HCN}$ and a lower ${ }^{14} \mathrm{~N} /{ }^{15} \mathrm{~N}$.

Indeed, other sources of $\mathrm{CN}$ - simple or complex organic molecules - have been mentioned long ago in the literature, notably $\mathrm{C}_{2} \mathrm{~N}_{2}$ (see, e.g., Swings \& Haser 1956).

The difficulties met when investigating $\mathrm{HCN}$ and $\mathrm{CH}_{3} \mathrm{CN}$ as possible parents of $\mathrm{CN}$ were raised by Bockelée-Morvan \& Crovisier (1985) on the basis of the expansion velocity in the coma. They suggested $\mathrm{C}_{2} \mathrm{~N}_{2}$ and $\mathrm{HC}_{3} \mathrm{~N}$ as possible alternatives. After re-examination of many near-UV observations of the $\mathrm{CN}$ radicals and high-dispersion spectra of the $\mathrm{CN}$ Violet (0-0) band in the coma of comet C/Austin 1989 X1, Festou et al. (1998) favor a single parent which is neither HCN nor $\mathrm{CH}_{3} \mathrm{CN}$, but a molecule having a lifetime of about $3.5 \times 10^{4} \mathrm{~s}$ at $1 \mathrm{AU}$. They propose the chemically stable $\mathrm{C}_{2} \mathrm{~N}_{2}$ as a likely candidate. Following the same lead, Bonev \& Komitov (2000) find that $\mathrm{C}_{2} \mathrm{~N}_{2}$ is indeed to be preferred over $\mathrm{HCN}$ as a single parent of $\mathrm{CN}$. A recent analysis of the $\mathrm{CN}$ profile in comet 21P/Giacobini-Zinner by Lara et al. (2003) showed that HCN should be ruled out as the sole parent of $\mathrm{CN}$. On the other hand, several analyses present $\mathrm{HCN}$ as the major or sole source of $\mathrm{CN}$ (e.g., Korsun \& Jockers 2002; Lara et al. 2004).

Fray et al. (2004) propose HCN polymers as a source of CN. A'Hearn et al. 2002 provide strong evidence that most $\mathrm{CN}$ are produced from grains in the coma rather than from nuclear ices. The link with CHON particles was suggested. However, the correlation between the dust and $\mathrm{CN}$ distribution in the comae is not perfect. This was shown by A'Hearn et al. (1986) in the case of comet P/Halley. In a study of comet Hale-Bopp, Woodney et al. (2004) find that there is a better correlation between $\mathrm{HCN}$ and $\mathrm{CN}$ than between $\mathrm{HCN}$ and the optically dominant dust. A dust source could then be small or/and low-albedo undetectable particles instead of the observable dust, like black HCN polymers (e.g. Rettig et al. 1992). A very dusty comet like Hale-Bopp shows the same isotopic characteristics as quasi dust-free comets (e.g., 122P/de Vico, see Jehin et al. 2004) and the latter do not show abnormally low CN production rates. Greenberg \& $\mathrm{Li}$ (1998) conclude that the source of $\mathrm{CN}$ and some other simple molecules is the organic component in comet dust.
The IDPs with elevated ${ }^{15} \mathrm{~N}$ point toward the presence of organic molecules, e.g., aromatic hydrocarbons (Keller et al. 2004). These may be related to the organic component of the cometary grains and explain the high ${ }^{15} \mathrm{~N}$ abundance (see also Arpigny et al. 2003).

The link between $\mathrm{CN}$ and $\mathrm{HCN}$ in cometary comae has generally been investigated by looking for similarities between scale lengths and between production rates. This yields different results in different comets, a variability which would show up in the isotopic ratios. The isotopic measurements provide an independent and powerful fingerprinting method. If confirmed, they would show that $\mathrm{HCN}$ is not the unique - and probably not the major - parent of $\mathrm{CN}$ at small as well as large heliocentric distances.

The origin of $\mathrm{CN}$ is still a puzzle, as is the apparent isotopic differentiation within cometary constituents and its consequences on our understanding of the early solar system. Additional determinations of the $\mathrm{N}$ isotopic ratio in $\mathrm{HCN}$ and other N-bearing molecules are highly desirable in order to shed light on these issues.

\section{References}

A’Hearn, M. F., Hoban, S., Birch, P. V., et al. 1986, Nature, 324, 649 A'Hearn, M. F., Millis, R. L., Schleicher, D. G., Osip, D. J., \& Birch, P. V. 2002, Icarus, 118, 223

Arpigny, C., Jehin, E., Manfroid, J., et al. 2003, Science, 301, 1522

Bockelée-Morvan, D., \& Crovisier, J. 1985, A\&A, 151, 90

Bonev, B., \& Komitov, B. 2000, AAS DPS, 32, 1072

Fray, N., Bénilan, Y., Cottin, H., et al. 2004, Meteoritics \& Planetary Science, 39, 581

Festou, M. C., Barale, O., Davidge, T., et al. 1998, AAS DPS, 30, 1089 Greenberg, J. M., \& Li, A. 1998, A\&A, 332, 374

Irvine, W. M., Dickens, J. E., Lovell, et al. 1998, Chemistry and Physics of Molecules and Grains in Space, Faraday Discussions, 109,475

Jewitt, D. C., Matthews, H. E., Owen, T., \& Meier, R. 1997, Science, 278, 90

Jehin, E., Manfroid, J., Cochran, A. L., et al. 2004, ApJ, 613, L161

Keller, L. P., Messenger, S., Flynn, G. J., et al. 2004, Geochim. Cosmochim. Acta, 68, 2577

Korsun, P. P., \& Jockers, K. 2002, A\&A, 381, 703

Lara, L.-M., Licandro, J., Oscoz, A., \& Motta, V. 2003, A\&A, 399, 763

Lara, L.-M., Tozzi, G. P., Boehnhardt, H., DiMartino, M., \& Schutz, R. 2004, A\&A, 422, 717

Rauer, H., Helbert, J., Arpigny, C., et al. 2003, A\&A, 397, 1109

Rettig, T. W., Tegler, S. C., Pasto, D. J., \& Mumma, M. J. 1992, ApJ, 398, 293

Swings, P., \& Haser, L. 1956, Atlas of Representative Comertary Spectra, Institut d'Astrophysique, Liège

Ziurys, L. M., Savage, C., Brewster, M. A., et al. 1999, ApJ, 527, L67

Woodney, L. M., A'Hearn, M. F., Schleicher, D. G., et al. 2002, Icarus, 157, 193 